\title{
Commissioning of the Silicon Strip Detector (SSD) of ALICE
}

\section{Panos Christakoglou for the ALICE Collaboration*}

$\dagger$

NIKHEF - Utrecht University

E-mail: Panos.Christakoglou@cern.ch

\begin{abstract}
The latest results from the commissioning of the SSD with cosmics are presented in this paper. The hardware status of the detector, the front-end electronics, cooling, data acquisition and issues related to the on-line monitoring are shown. In addition, the procedures implemented and followed to address the alignment with the rest of the ITS sub-detectors along with both on-line and off-line calibration strategies are described. Finally, results from simulations as well as from the reconstruction of cosmic data demonstrating the performance of the detector are presented, proving that the SSD is ready for the forthcoming proton-proton data taking.
\end{abstract}

The 2009 Europhysics Conference on High Energy Physics,

July 16 - 222009

Krakow, Poland

\footnotetext{
* Speaker.

$\dagger$ The author acknowledges the valuable contributions of Marek Chojnacki, Andrea Dainese, Marco van Leeuwen, Francesco Prino and Andrea Rossi. Special thanks go to the ALICE SSD Collaboration.
} 


\section{Introduction}

The Inner Tracking System (ITS) of the ALICE experiment [1], consists of six cylindrical layers of silicon detectors, the Silicon Pixel Detectors (SPD), the Silicon Drift Detectors (SDD) and the Silicon Strip Detectors (SSD). The outer layers are made of double sided Silicon Strip Detectors mounted on carbon-fiber support structures [1]. The SSD is crucial for the connection of tracks from the main tracking device of ALICE, the Time Projection Chamber (TPC), to the ITS and also provides $\mathrm{dE} / \mathrm{dx}$ information to assist particle identification for low-momentum particles [设]. The detector consists of 1698 modules each one having $768 \mathrm{P}$ - and $768 \mathrm{~N}$-side strips, resulting in total to more than 2.6 million channels. The SSD has been actively participating in all the commissioning and run activities as well as in all the data taking periods of the ALICE experiment.

\section{Commissioning results}

The installation of the entire ITS in its final position took place in June 2007. During the first commissioning phase (July - October 2007), all the connections were checked in detail. In December 2007 and in February/March 2008, the SSD participated in the first and second cosmics run respectively, during which partial cooling was available. As a consequence only a small fraction of the detector was included in the data acquisition system. The installation of the services and the upgrade of the cooling plant took place in May 2008, allowing us to include all the SSD modules in the third cosmics run that started in June 2008. The scope of this run was to collect a suitable data sample to perform the first part of the detector's alignment and charge calibration. The following paragraphs will summarize the results obtained during the summer of 2008.

\subsection{Detector operation}

During the cosmics run 1477 out of 1698 SSD modules took data in summer 2008. The fraction of bad strips was $\approx 1.5 \%$. Most of the modules not included in the data taking were drawing unexpected high bias current and were switched off as a precaution, pending further investigation, although their performance was still good. The resulting signal over noise ratio was better than $S / N>40$. Figure 1 shows the distribution in z (along the beam axis) and $\phi$ (azimuthal angle) of the reconstructed clusters for both SSD layers. The SPD FastOR [1] was used as a trigger detector, the rate of which reached the value of $\approx 0.18 \mathrm{~Hz}$.

\subsection{Alignment}

The study of the displacements and deformations of the SSD modules enhances the knowledge of the realistic detector geometry and thus contributes to the tracking performance. This is performed using cosmic data as well as pp events when the latter will be available. The starting point of the alignment procedure is the optical measurement (survey) performed during the construction. Then different tools are used in order to extract the relevant information. This allows us to align sensitive elements mounted on common mechanical supports (in the SSD case these are the 72 ladders), whereas with higher statistics we will be able to move to the level of a single module. Figure 2-left shows the distributions of the distances in $r-\phi$ between the track fitted on the outer SSD layer and the points measured in the inner SSD layer. The inclusion of the survey 


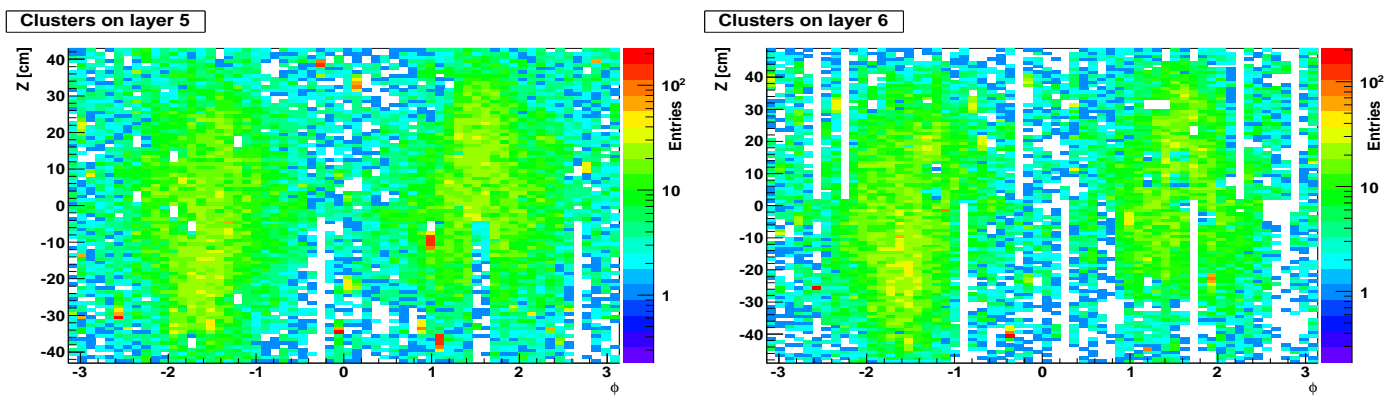

Figure 1: The distribution of reconstructed clusters from the cosmic data taking for layer 5 (left) and layer 6 (right) as a function of the azimuthal angle $\phi$ and the global $\mathrm{z}$ coordinate (along the beam axis).
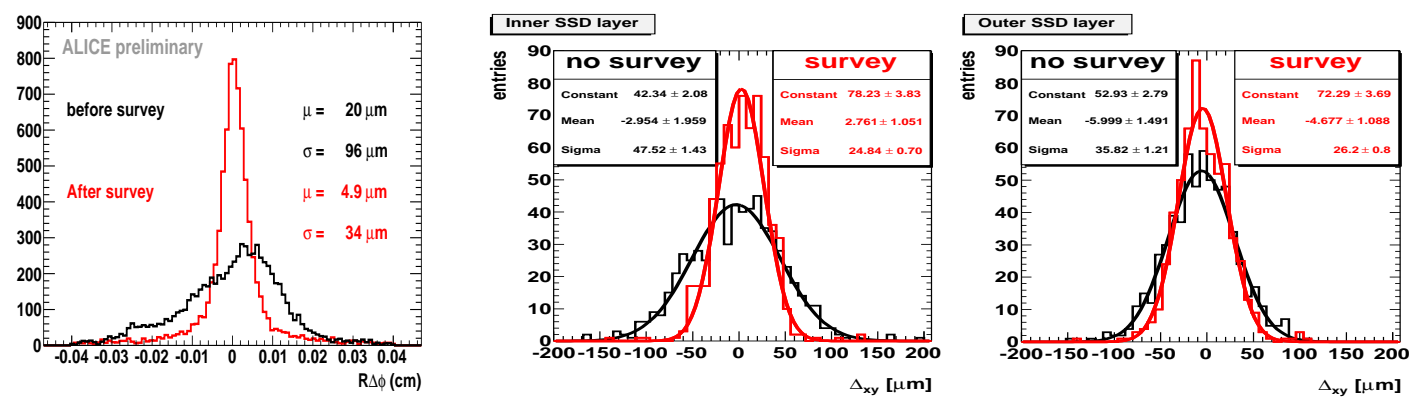

Figure 2: Track to point residuals with and without the SSD survey information (left plot). Track to extra cluster $\Delta x y$ for the two SSD layers (middle plot: layer 5, right plot: layer 6) before and after the usage of the SSD survey data.

data reduces significantly not only the mean of the distributions (from $20 \mu \mathrm{m}$ to $4.9 \mu \mathrm{m}$ ) but also its spread (from $96 \mu \mathrm{m}$ to $34 \mu \mathrm{m}$ ). In addition, the middle and right plots of fig. 2 show the track to point distance for the SSD extra clusters in the $r \phi$ plane without and with the inclusion of the survey data for the inner and outer SSD layers respectively. The extra clusters are created when particles cross a region with an acceptance overlap between two adjacent modules. In both cases, the inclusion of the SSD survey data improves the extracted resolution by a factor of $\approx 1.9$ and $\approx 1.3$ for the two layers [3].

\subsection{Charge calibration}

The gain calibration of the SSD has two components: relative calibration of the $\mathrm{P}$ and $\mathrm{N}$ sides and overall calibration of ADC values to energy loss. The charge matching is a strong point of double sided silicon sensors and helps to remove fake clusters. Both calibrations relied on cosmics. Already in the laboratory the calibration constants were determined, using cosmics on a spare ladder using a data-acquisition system setup which matches the one used in the experiment. These constants were refined during the cosmics runs with the pixel trigger.

For the charge matching, we relied on runs without the presence of the magnetic field. Only modules with large number of accumulated cluster statistics were considered. Corrections were applied to get the best calibration results at the module level. The resulting normalized difference in P- and N-charge, as illustrated in fig. B-left, has a FWHM of $11 \%$ [4]. Detailed studies were 

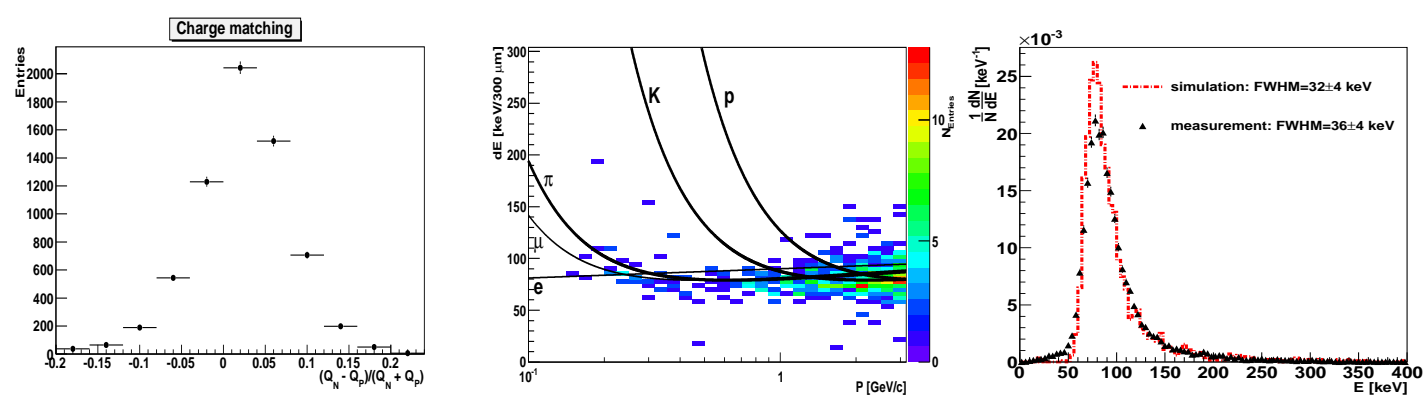

Figure 3: The distribution of the normalized difference in $\mathrm{P}$ - and $\mathrm{N}$ - charge (left). The dE/dx distributions as a function of the particle's momenta for cosmic data (middle). The charge distribution for both simulations and cosmic data (right).

performed to check the calibration constants at the chip level though the accumulated statistics was not sufficient to extract a definite conclusion. Preliminary results show that the calibration at the chip level improves the stability of the P-and N-side gains by $10 \%$ [ [

For the absolute calibration, the analysis of cosmic data obtained with the magnetic field was used. Due to the fact that the TPC calibration was not optimized and resulted in a poor ITS-TPC track matching, we mainly relied on the stand-alone ITS tracking [2]. Corrections were applied to take into account the inclination of the track, thus addressing the issue of the different track lengths when a particle crosses a module.

Figure 3 -middle shows the energy loss measured by the two SSD layers as a function of the particles' momenta for the cosmic data sample analyzed. The curves are drawn based on [5]. Figure 3 -right gives the comparison of the charge distributions for both simulated and real data. The FWHM obtained for the two distributions differ by $12.5 \%$.

\section{Summary}

In this paper we presented the main results from the commissioning of the SSD of ALICE using cosmic data. The SSD participated with great efficiency in the different ALICE commissioning periods started in December 2007. The successful cosmics data taking in summer 2008, allowed us to perform the first part of the detector's alignment and calibration. The data that are going to be collected in the upcoming period will be used for the further refinement of these two activities. In conclusion, the SSD performance results are very close to the designed specifications and is ready for the first $\mathrm{pp}$ and $\mathrm{Pb}-\mathrm{Pb} \mathrm{LHC}$ collisions.

\section{References}

[1] K. Aamodt et al. [ALICE Collaboration], JINST 3, (2008) S08002.

[2] B. Alessandro et al. [ALICE Collaboration], J. Phys. G30, (2004) 1517.

[3] A. Dainese, A. Rossi, M, van Leeuwen, N. Vermeer, ALICE Internal Note in preparation.

[4] M. Chojnacki, ALICE Internal Note in preparation.

[5] H. Bichsel, NIM A562, (2006) 154. 